\title{
Evidence from a meta-analysis: is nivolumab neurotoxic in cancer patients?
}

This article was published in the following Dove Press journal:

OncoTargets and Therapy

I March 2017

Number of times this article has been viewed

\section{Xiangyi Kong ${ }^{1,2}$ \\ Yanguo Kong'}

'Department of Neurosurgery, Peking Union Medical College Hospital, Chinese Academy of Medical Sciences, Dongcheng District, Beijing, People's Republic of China; ${ }^{2}$ Department of Anesthesia, Critical Care and Pain Medicine, Massachusetts General Hospital, Harvard Medical School, Harvard University, Boston, MA, USA

Correspondence: Yanguo Kong Department of Neurosurgery, Peking Union Medical College Hospital, Chinese Academy of Medical Sciences, No I Shuaifuyuan, Dongcheng District, Beijing 100730, People's Republic of China Email kong0126@I26.com

\begin{abstract}
The aim of this study was to summarize the findings of previous studies focusing on whether the risks of certain neurotoxicities are correlated to the programmed death 1 (PD-1) inhibitor nivolumab versus other chemotherapy or immunotherapy drugs. Six eligible studies, including 3,023 patients, were considered in the meta-analysis. The risk ratios (RRs) of fatigue, headache, dysgeusia, vertigo, paresthesia, anxiety or malaise and peripheral neuropathy were 0.908 (95\% confidence interval [95\% CI]: 0.724, 1.138; $P=0.402), 0.841$ (95\% CI: 0.606, 1.168; $P=0.302), 0.423$ (95\% CI: 0.132, 1.357; $P=0.148), 0.762$ (95\% CI: $0.475,1.223 ; P=0.261$ ), 0.411 (95\% CI: $0.232,0.730 ; P=0.002), 1.049$ (95\% CI: 0.094, 11.752; $P=0.969)$ and 0.192 (95\% CI: $0.039,0.935 ; P=0.041)$, respectively. Our analysis supported that the PD-1 inhibitor nivolumab did not cause increased or decreased risks of fatigue, headache, dysgeusia, vertigo and anxiety or malaise and was associated with decreased risks of paresthesia and peripheral neuropathy as compared with controls. These outcomes indicated that although clinicians should be attentive of the side effects of nivolumab, in terms of nervous system side effects, nivolumab is generally safe.
\end{abstract}

Keywords: PD-1 inhibitor, nivolumab, neurotoxicity, cancer

\section{Introduction}

Immunotherapy has been considered to be one of the most important breakthroughs in cancer treatments in the last decade. Immune checkpoint inhibitors against cytotoxic T lymphocyte antigen-4 (CTLA-4) and programmed death 1 (PD-1) topped the list of the most noticeable and encouraging cancer therapies. ${ }^{1,2}$ As PD-1 is able to limit cytokine secretion, function and proliferation of T-cells, especially in cancer-bearing hosts or chronic viral infections, PD-1 inhibitors have been developed and have achieved many achievements. ${ }^{3}$ On the surface of tumor-infiltrating T-cells and circulating T-cells from melanoma patients, high levels of PD-1s have been tested, indicating that PD-1 inhibition might prevent cancer-correlated exhaustion of T-cells. ${ }^{4}$ The first batch of PD-1-inhibiting agents includes pembrolizumab (also named lambrolizumab) and nivolumab. Nivolumab, scientifically named BMS-936558/MDX-1106, is a fully human immunoglobulin G4 (IgG4) monoclonal PD-1-blocking antibody that abrogates its interaction with PD-L1 and PD-L2. ${ }^{5}$ In several of Phase III studies regarding nonsmall-cell lung cancer (NSCLC), renal cancer and melanoma, nivolumab administration has been shown to generate survival benefits. Because of this, the US Food and Drug Administration (FDA) approved its marketing in $2015 .^{6}$

Unlike traditional antineoplastic chemotherapies, nivolumab can potentially lead to some side effects, such as gastrointestinal, hepatotoxic, orthopedic and mucocutaneous toxicities. ${ }^{7}$ However, the incidence of nervous system adverse effects among 
clinical studies (such as fatigue, headache, dysgeusia, vertigo, paresthesia, anxiety or malaise and peripheral neuropathy) has shown a great variation. ${ }^{8-13}$ Until now, there are no comprehensive reviews and analyses to synthesize these data; thus, the overall neurotoxicity risks caused by nivolumab need to be further clarified. We performed this meta-analysis of randomized clinical studies to assess the overall risks of neurotoxicities in cancer patients receiving nivolumab as the anticancer therapy.

\section{Methods}

\section{Overview of systematic literature review}

We performed a systematic literature review to identify all studies that detailed PD-1 inhibitor nivolumab with its toxic and side effects.

\section{Search strategy and study selection}

In accordance with the Preferred Reporting Items for Systematic Reviews and Meta-Analyses (PRISMA) statement, 2 authors independently searched the databases Embase, PubMed, Chinese National Knowledge Infrastructure (CNKI) and Web of Knowledge (time: approximately September 2016) to enroll studies that met the inclusion criteria. The search term was "nivolumab". Search results were limited to randomized clinical studies without language limitations. If there were duplicate studies, only the most updated and complete ones were adopted. If there were disagreements between the 2 investigators, a discussion would be carried out or a third investigator would intervene. Inclusion criteria and exclusion criteria are listed in Table 1 . We also

Table I Inclusion and exclusion criteria for study selection in this meta-analysis

\begin{tabular}{|c|c|}
\hline Number & Inclusion criteria \\
\hline I & $\begin{array}{l}\text { Randomized Phase II and III studies in patients with } \\
\text { solid tumors. }\end{array}$ \\
\hline 2 & $\begin{array}{l}\text { The outcome of the study includes neurotoxicities such } \\
\text { as fatigue, headache, dysgeusia, vertigo, paresthesia, } \\
\text { anxiety or malaise and peripheral neuropathy. }\end{array}$ \\
\hline 3 & Participants received treatment with nivolumab. \\
\hline 4 & $\begin{array}{l}\text { The RR with a } 95 \% \mathrm{Cl} \text { of the risk of selected } \\
\text { neurotoxicities associated with nivolumab could be } \\
\text { obtained from articles directly or calculated based } \\
\text { on the figures or tables given in articles or through } \\
\text { contacting the authors. }\end{array}$ \\
\hline \multirow[t]{2}{*}{5} & $\begin{array}{l}\text { For the duplicate articles, only the most complete or } \\
\text { the most newly published one was included. }\end{array}$ \\
\hline & Exclusion criteria \\
\hline 1 & Phase I trials were excluded. \\
\hline 2 & $\begin{array}{l}\text { The treatments in experimental groups should not } \\
\text { include other drugs. }\end{array}$ \\
\hline
\end{tabular}

Abbreviations: $\mathrm{Cl}$, confidence interval; $\mathrm{RR}$, risk ratio. reviewed related references to identify other potentially eligible studies.

\section{Data extraction and clinical end points}

We retrieved the data independently. We gathered the following data: author name, publication year, phase of the trial, total number of patients enrolled for analysis, number of events for neurotoxicities (fatigue, headache, dysgeusia, vertigo, paresthesia, anxiety or malaise and peripheral neuropathy) and treatment arms. Jadad score scale was used to evaluate the methodological qualities of these studies (Table 2).

Any discrepancies between us were resolved by consensus. The common terminology criteria of adverse events (CTCAE, version 4.0) were used to record the toxicities and their grades in the included clinical trials. ${ }^{14}$ In these criteria, Grade 1 means mild, asymptomatic or mild symptoms, clinical or diagnostic observations only, intervention not indicated; Grade 2 means moderate, minimal, local or noninvasive intervention indicated, limiting age-appropriate instrumental activities of daily living (preparing meals, shopping for groceries or clothes, using the telephone, managing money, etc); Grade 3 means severe or medically significant but not immediately life-threatening, hospitalization or prolongation of hospitalization indicated, disabling, limiting self-care activities of daily living (bathing, dressing and undressing, feeding self, using the toilet, taking medications and not bedridden); Grade 4 means life-threatening consequences, urgent intervention indicated; and Grade 5 means death related to adverse effects.

\section{Data synthesis}

Differences were expressed as risk ratios (RRs) with 95\% confidence intervals (95\% CIs). The Labbe plot, $I^{2}$ test and Cochran's Q-test (Table 3) were conducted to estimate the heterogeneity among the studies. ${ }^{15,16}$ If no evidence of statistical heterogeneity was detected, we chose to use a fixed-effects model. Otherwise, the random-effects model of Laird and DerSimonian was adopted. To assess the stability of the pooled values, we also performed sensitivity analyses (Table 3). ${ }^{15}$ Using contour-enhanced funnel plots (Table 3), potential publication biases were estimated. Since only 6 studies were included, metaregression analyses were not performed.

The $P$-value $<0.05$ was deemed as statistical significance. The statistical analysis was done through Review Manager 5.3 (The Cochrane Collaboration, Oxford, UK) and STATA 12.0 (StataCorp LP, College Station, TX, USA) software. 


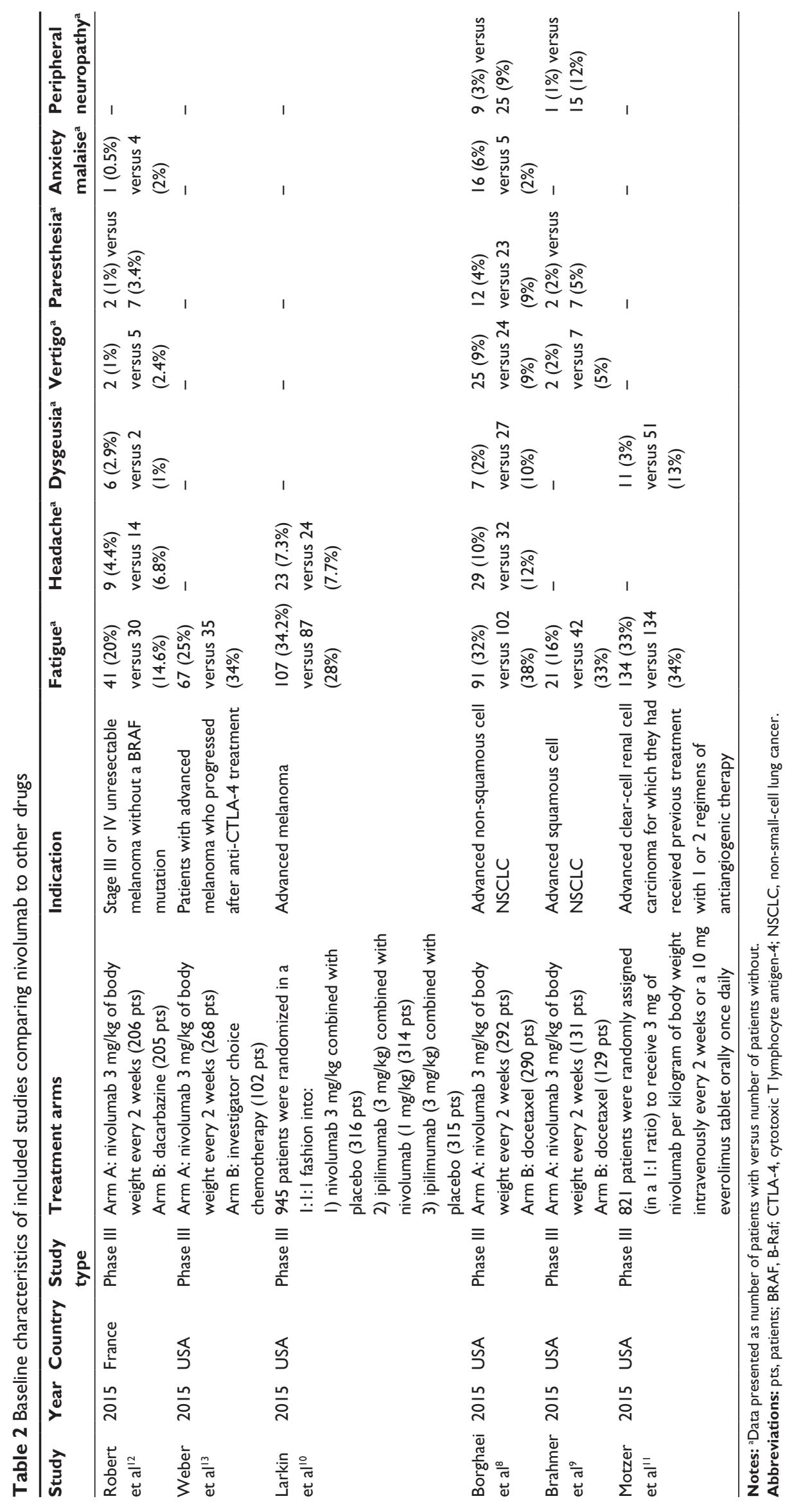


Table 3 The statistical methods used in this meta-analysis and their explanation

\begin{tabular}{|c|c|c|}
\hline Statistical means & Goals and usages & Explanation \\
\hline Labbe plot & $\begin{array}{l}\text { To evaluate heterogeneity } \\
\text { between the included studies }\end{array}$ & $\begin{array}{l}\text { In Labbe figure, if the points basically present as a linear distribution, it } \\
\text { can be taken as an evidence of homogeneity. }\end{array}$ \\
\hline Cochran's $Q$ test & $\begin{array}{l}\text { To evaluate heterogeneity } \\
\text { between the included studies }\end{array}$ & $\begin{array}{l}\text { Cochran's } Q \text { test is an extension to the McNemar test for related } \\
\text { samples that provides a method for testing for differences between } 3 \text { or } \\
\text { more matched sets of frequencies or proportions. Heterogeneity was } \\
\text { also considered significant if } P<0.05 \text { using the Cochran's } Q \text { test. }\end{array}$ \\
\hline$P^{2}$ index test & $\begin{array}{l}\text { To evaluate heterogeneity } \\
\text { between the included studies }\end{array}$ & $\begin{array}{l}\text { The } l^{2} \text { index measures the extent of true heterogeneity dividing the } \\
\text { difference between the result of the } \mathrm{Q} \text { test and its degrees of freedom } \\
(\mathrm{k}-\mathrm{I}) \text { by the } \mathrm{Q} \text { value itself and multiplied by } 100 . I^{2} \text { values of } 25 \%, 50 \% \\
\text { and } 75 \% \text { were used as evidence of low, moderate and high heterogeneity, } \\
\text { respectively. }\end{array}$ \\
\hline Sensitivity analysis & $\begin{array}{l}\text { To examine the stability of } \\
\text { the pooled results }\end{array}$ & $\begin{array}{l}\text { A sensitivity analysis was performed using the one-at-a-time method, } \\
\text { which involved omitting one study at a time and repeating the meta- } \\
\text { analysis. If the omission of one study significantly changed the result, it } \\
\text { implied that the result was sensitive to the studies included. }\end{array}$ \\
\hline $\begin{array}{l}\text { Contour-enhanced } \\
\text { funnel plot }\end{array}$ & Publication bias test & $\begin{array}{l}\text { Visual inspection of the contour-enhanced funnel plots was used to assess } \\
\text { potential publication bias. Asymmetry in the plots, which may be due } \\
\text { to studies missing on the left-hand side of the plot that represents low } \\
\text { statistical significance, suggested publication bias. If studies were missing } \\
\text { in the high statistical significance areas (on the right-hand side of the plot), } \\
\text { the funnel asymmetry was not considered to be due to publication bias. }\end{array}$ \\
\hline
\end{tabular}

\section{Results}

\section{Search results and characteristics of the studies}

The article search process was conducted as shown in Figure 1. A total of 6 articles involving 3,023 patients finally met the inclusion criteria. ${ }^{8-13}$ All the 6 studies were written in English and were randomized Phase III trials. Five of the 6 studies $^{8-11,13}$ were carried out in the US and 1 in France. ${ }^{12}$ Their baseline characteristics, including treatment arms, interventions and clinical indications, are shown in Table 2.

\section{Quality of the included studies}

Table 4 shows the different elements of the Jadad scale for all the included studies in terms of blinding, randomization, an account of all patients and the overall score.

\section{Meta-analysis regarding nivolumab and neurotoxicity}

Fatigue was reported in all the 6 studies, ${ }^{8-13}$ headache, ${ }^{8,10,12}$ dysgeusia, ${ }^{8,11,12}$ vertigo $^{8,9,12}$ and paresthesia ${ }^{8,9,12}$ were reported in 3 of the 6 studies; anxiety or malaise ${ }^{8,12}$ and peripheral neuropathy ${ }^{8,9}$ were, respectively, reported in 2 studies. RRs of all-grade neurotoxic events were calculated on the basis of the included studies. For example, in the case of fatigue, from the Labbe figure (Figure 2A), the points did not present as a linear distribution, which can be taken as evidence of heterogeneity among the included studies $(\mathrm{Q}=18.47, d f=5$, $\left.I^{2}=72.9 \%, P=0.002\right)$. Consequently, a random-effects model was utilized to summarize the data. As revealed in Figure 3A, the forest plot demonstrated that nivolumab administration did not increase or decrease the fatigue risk (RR 0.908, 95\% CI $0.724,1.138 ; P=0.402$ ).

We also tested the correlation between the risks of headache, dysgeusia, vertigo, paresthesia, anxiety or malaise and peripheral neuropathy and nivolumab administration (versus dacarbazine, docetaxel, ipilimumab or everolimus according to the effect models) (headache: $\mathrm{Q}=0.63, d f=2, I^{2}=0.0 \%$, $P=0.730$, fixed-effects model, Figure $2 \mathrm{~B}$; dysgeusia: $\mathrm{Q}=9.42$, $d f=2, I^{2}=78.8 \%, P=0.009$, random-effects model, Figure $2 \mathrm{C}$; vertigo: $\mathrm{Q}=2.99, d f=2, I^{2}=33.2 \%, P=0.224$, fixed-effects model, Figure 2D; paresthesia: $\mathrm{Q}=0.68, d f=2, I^{2}=0.0 \%$, $P=0.710$, fixed-effects model, Figure $2 \mathrm{E}$; anxiety or malaise: $\mathrm{Q}=4.17, d f=1, I^{2}=76.0 \%, P=0.041$, random-effects model, Figure 2F; peripheral neuropathy: $\mathrm{Q}=2.42, d f=1, I^{2}=58.7 \%$, $P=0.120$, random-effects model). The results also suggest no significant differences in terms of headache (RR 0.841 , 95\% CI 0.606, 1.168; $P=0.302$; Figure 3B), dysgeusia (RR 0.423, 95\% CI 0.132, 1.357; $P=0.148$; Figure 3C), vertigo (RR 0.762, 95\% CI 0.475, 1.223; $P=0.261$; Figure 3D) and anxiety or malaise (RR 1.049, 95\% CI 0.094, 11.752; $P=0.969$; Figure 3F). In addition, regarding paresthesia and peripheral neuropathy, nivolumab was even statistically associated with decreased risks (paresthesia: RR 0.411, 


\section{PRISMA 2009 flow diagram}

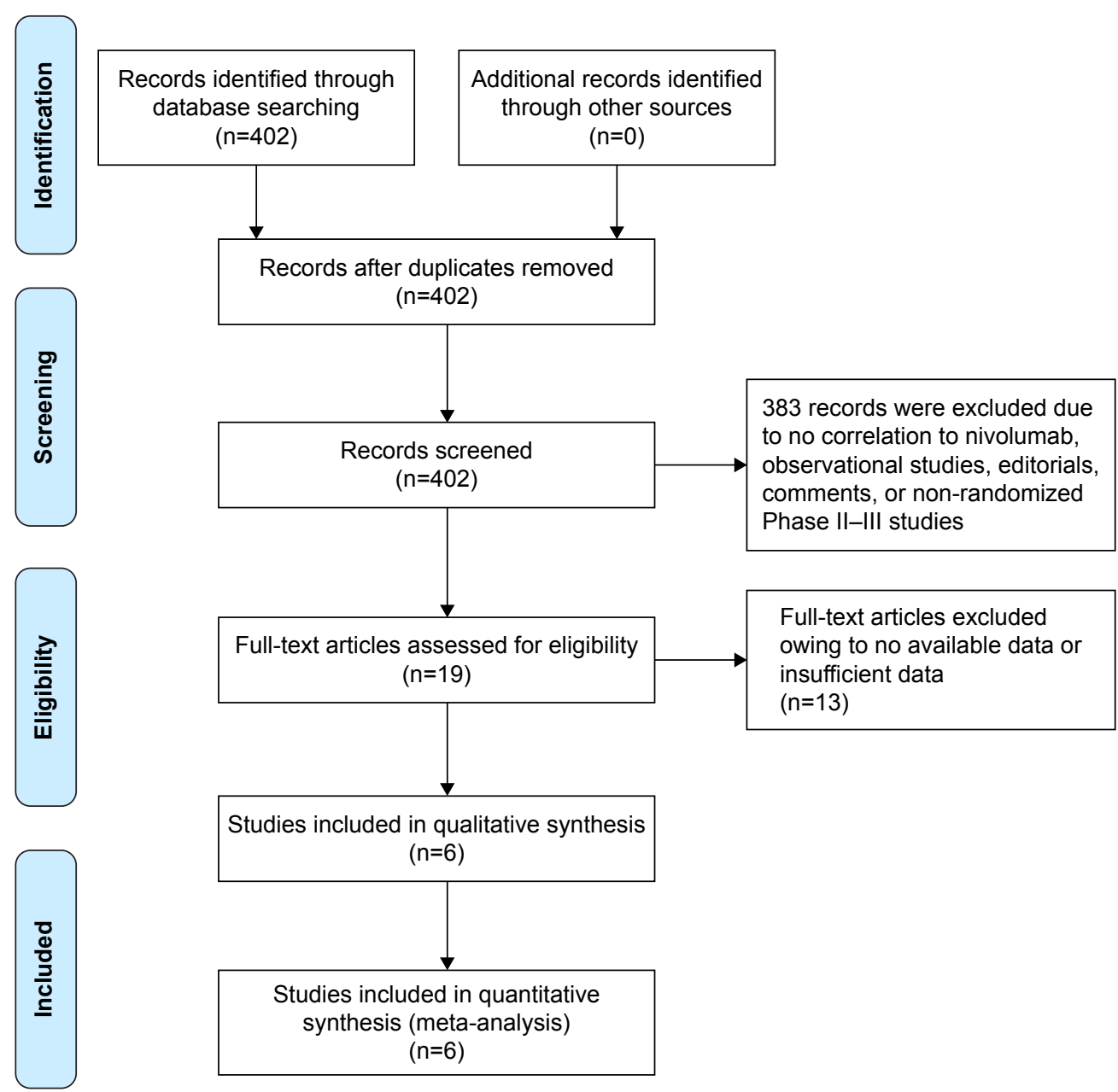

Figure I Literature search and selection of articles.

Note: Adapted from Moher D, Liberati A, Tetzlaff J, Altman DG, The PRISMA Group (2009) Preferred Reporting Items for Systematic Reviews and Meta-Analyses: The PRISMA Statement. PLoS Med 6(7): el 000097. Creative Commons license and disclaimer available from: http://creativecommons.org/licenses/by/4.0/legalcode. ${ }^{20}$

Abbreviation: PRISMA, Preferred Reporting Items for Systematic Reviews.

95\% CI 0.232, 0.730; $P=0.002$; Figure 3E; peripheral neuropathy: RR $0.192,95 \%$ CI $0.039,0.935 ; P=0.041$; Figure 3G).

\section{Sensitivity analysis and publication bias}

To assess whether a single study could affect the final RRs, each individual study was removed one time and the data were re-pooled. The analysis results demonstrated that the pooled RRs were not affected by deleting every single study (Figure 2G-K). The contour-enhanced funnel plots were employed to reveal the publication bias, showing that the studies had missing areas for high statistical significance (in the right-hand side of the plot), indicating that there was no publication bias in the study (Figure 2L-P).

Table 4 Jadad quality assessment of the included studies

\begin{tabular}{llllllll}
\hline Study & Year & Country & Study type & Randomization & Blinding & An account of all patients & Overall score \\
\hline Robert et al $^{12}$ & 2015 & France & Phase III & 2 & 2 & I & 5 \\
Weber et al $^{13}$ & 2015 & USA & Phase III & 2 & 0 & I & 3 \\
Larkin et al $^{10}$ & 2015 & USA & Phase III & 2 & 2 & I & 3 \\
Borghaei et al $^{8}$ & 2015 & USA & Phase III & 2 & 0 & I & 3 \\
Brahmer et al $^{9}$ & 2015 & USA & Phase III & 2 & 0 & I & 3 \\
Motzer et al $^{\prime \prime}$ & 2015 & USA & Phase III & 2 & 0 & I & 3 \\
\hline
\end{tabular}




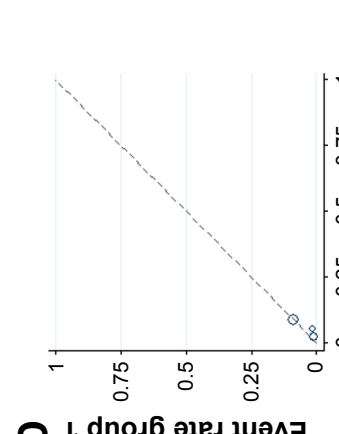

อ $レ$ dnoג6 әред ұиәл

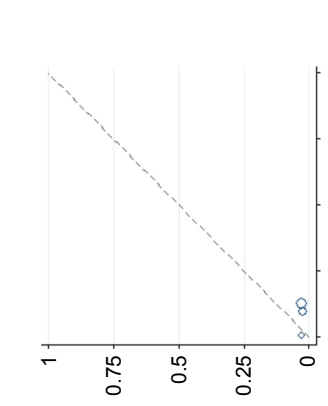

U ᄂ dnoג6 әүед ұиәл

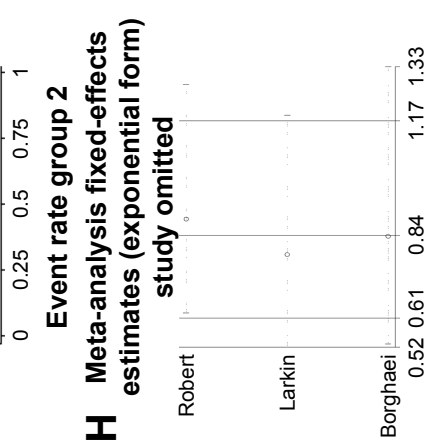

I

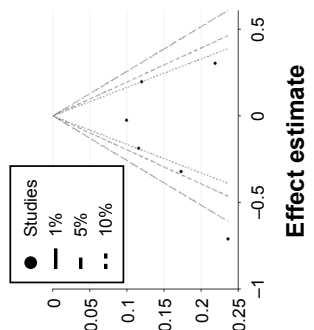

лодлә рлерuетs
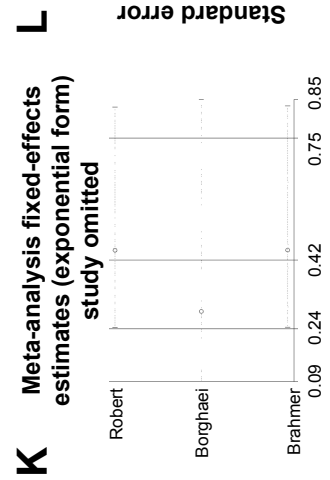

口

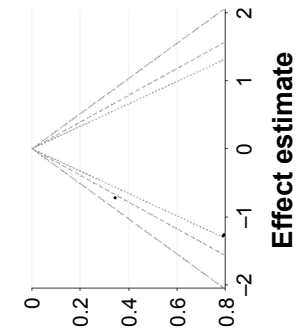

lodsa psepuets

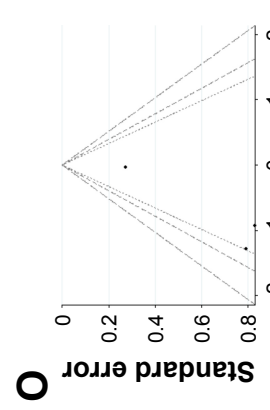

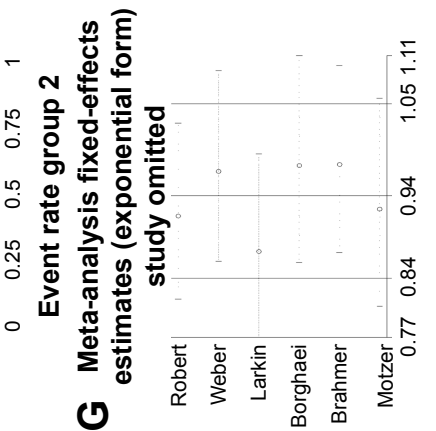

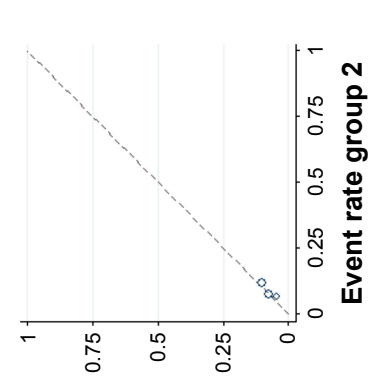

m ldnoג6 әред ұиәк $\exists$

ᄂ ᄂ Inod6 әэед ұиәм
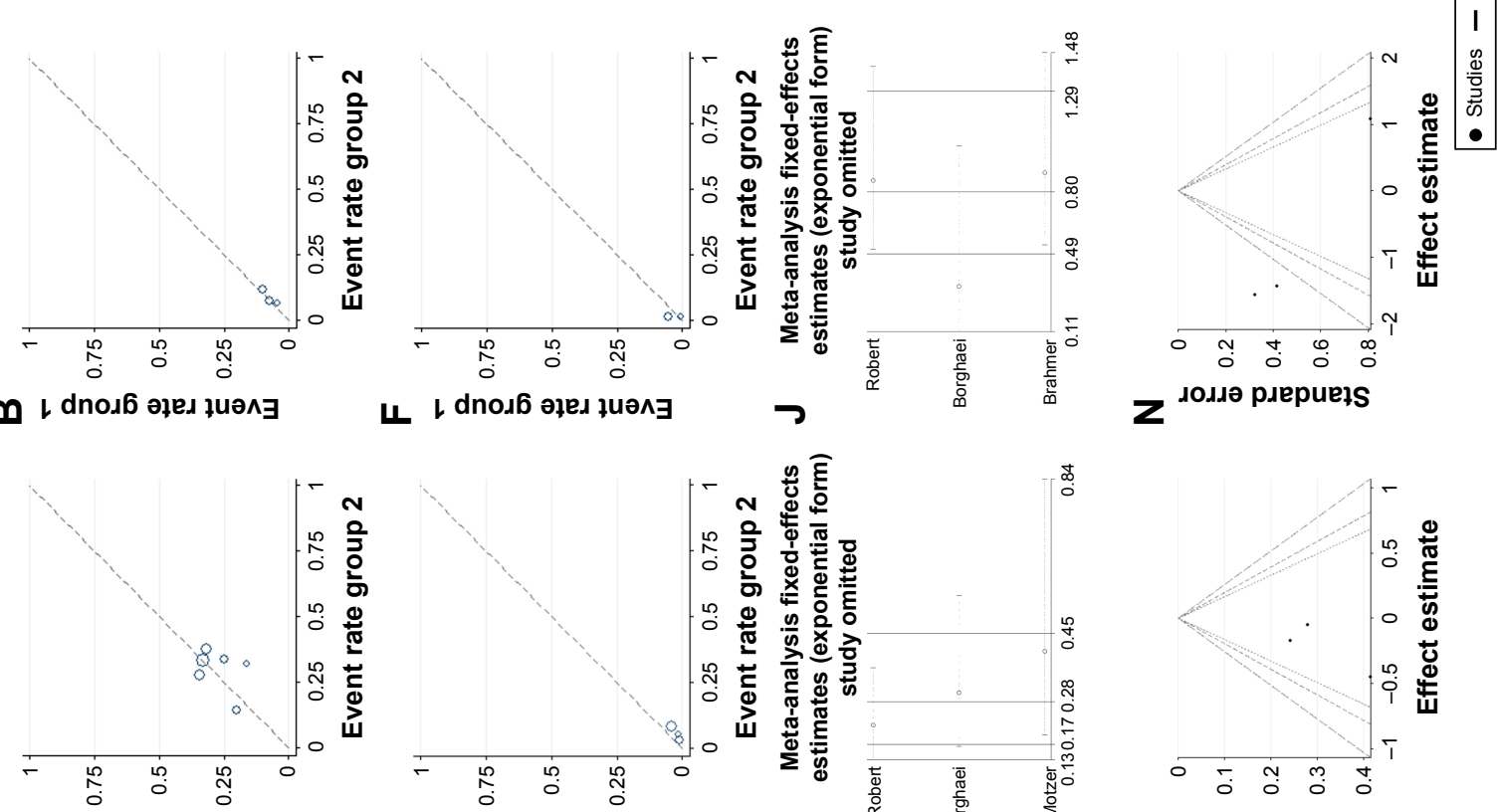

$\varangle\llcorner$ dnoג6 әұед ұиәлэ
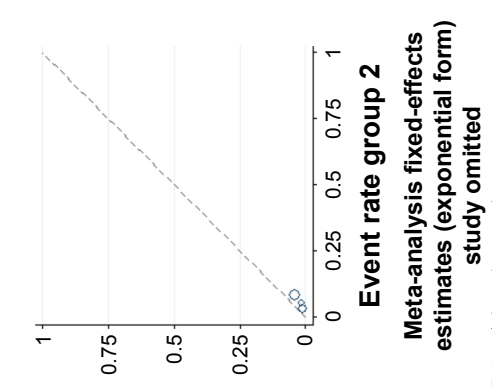

ш $レ$ dnoג6 әред ұиәкヨ
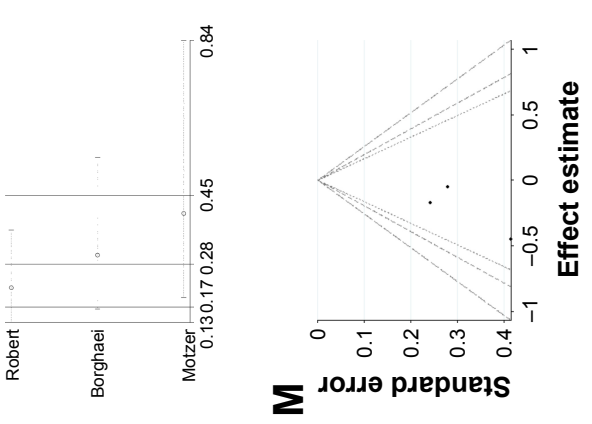

$\Sigma$

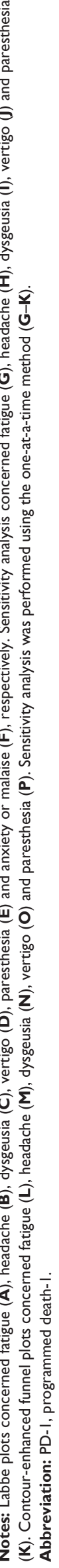


A

Forest plot of relative risk (RR) of fatigue associated with nivolumab versus control

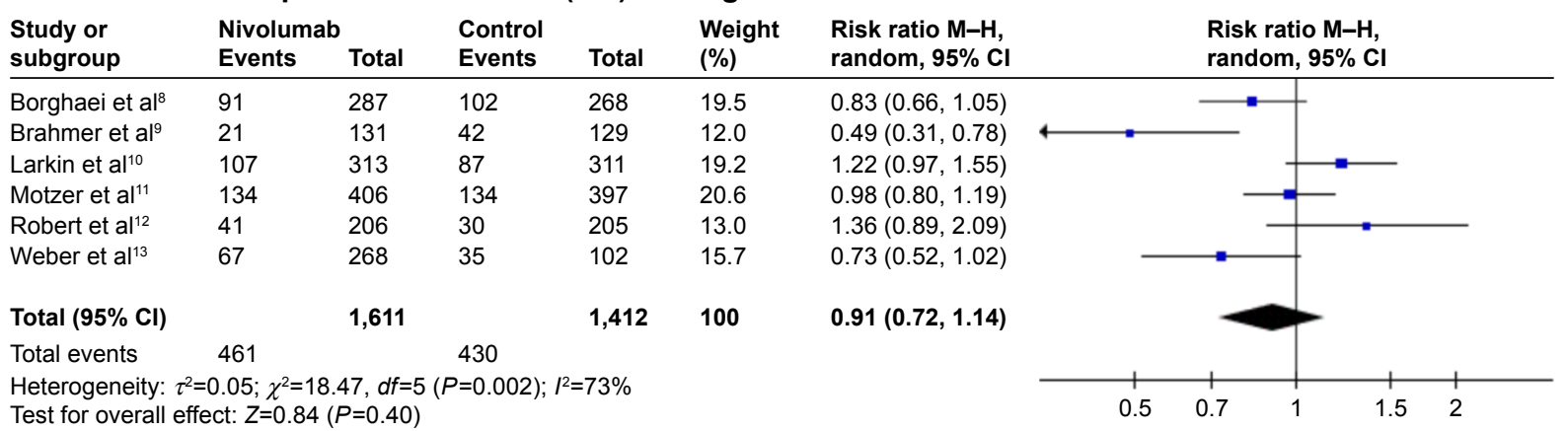

B Forest plot of relative risk (RR) of headache associated with nivolumab versus control

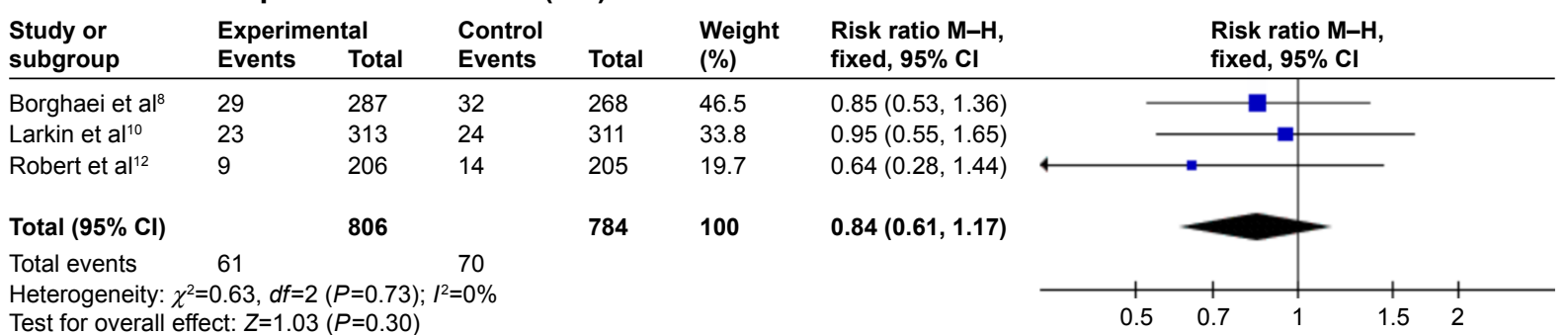

C Forest plot of relative risk (RR) of dysguesia associated with nivolumab versus control

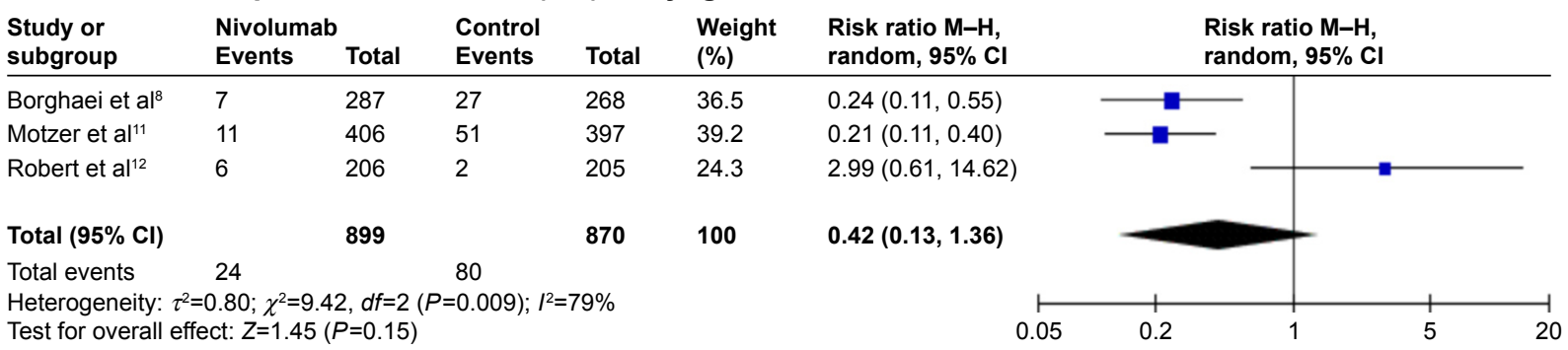

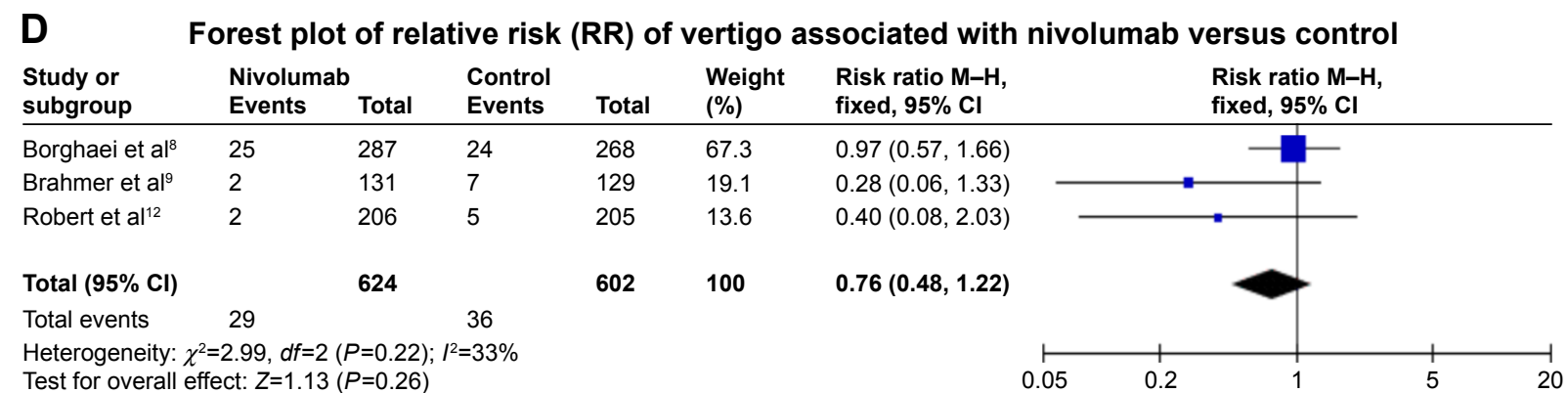

E Forest plot of relative risk (RR) of paresthesia associated with nivolumab versus control

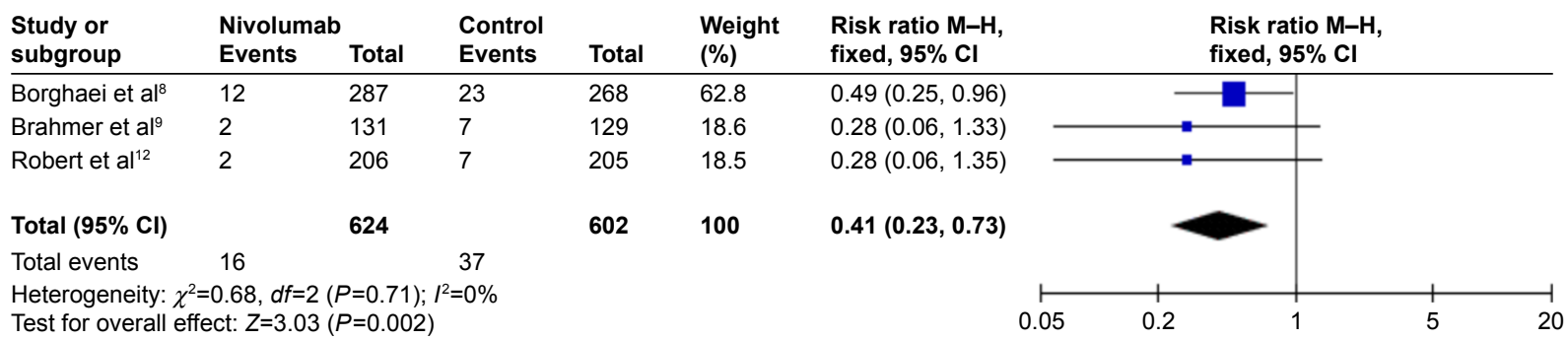

Figure 3 (Continued) 
F Forest plot of relative risk (RR) of anxiety or malaise associated with nivolumab versus control

\begin{tabular}{|c|c|c|c|c|c|c|}
\hline $\begin{array}{l}\text { Study or } \\
\text { subgroup }\end{array}$ & $\begin{array}{l}\text { Nivolumab } \\
\text { Events }\end{array}$ & Total & $\begin{array}{l}\text { Control } \\
\text { Events }\end{array}$ & Total & $\begin{array}{l}\text { Weight } \\
(\%)\end{array}$ & $\begin{array}{l}\text { Risk ratio } \mathrm{M}-\mathrm{H} \text {, } \\
\text { random, } 95 \% \mathrm{Cl}\end{array}$ \\
\hline Borghaei et $\mathrm{al}^{8}$ & 16 & 287 & 5 & 268 & 57.9 & $2.99(1.11,8.04)$ \\
\hline Robert et al ${ }^{12}$ & 1 & 206 & 4 & 205 & 42.1 & $0.25(0.03,2.21)$ \\
\hline Total $(95 \% \mathrm{Cl})$ & & 493 & & 473 & 100 & $1.05(0.09,11.75)$ \\
\hline Total events & 17 & & 9 & & & \\
\hline $\begin{array}{l}\text { Heterogeneity: } \\
\text { Test for overall }\end{array}$ & $\begin{array}{l}2.37 ; \chi^{2}=4.1 \\
z=0.04\end{array}$ & $\begin{array}{l}\text { 7, } d f=1 \\
P=0.97\end{array}$ & & & & 0.02 \\
\hline
\end{tabular}

G Forest plot of relative risk (RR) of peripheral neuropathy associated with nivolumab versus control

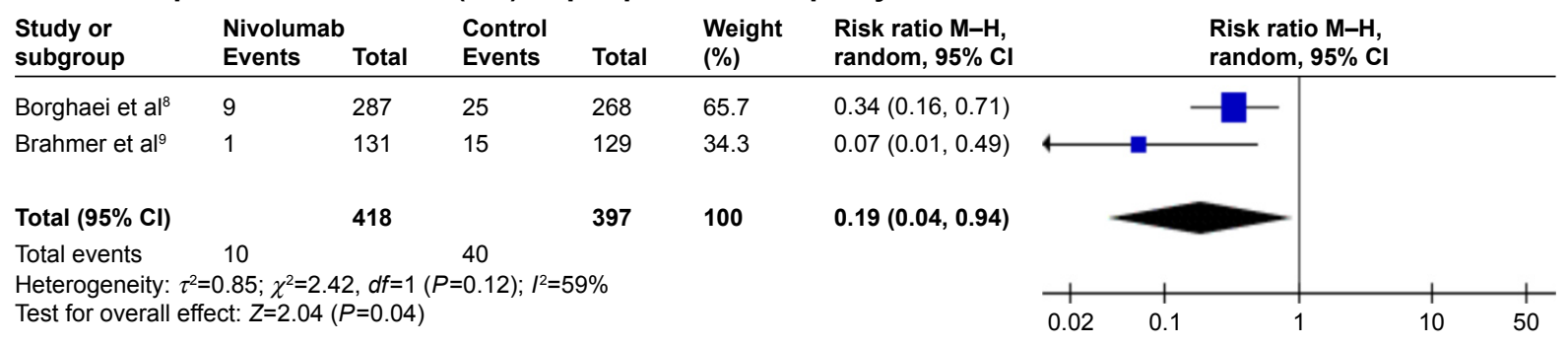

Figure 3 Forest plots (individual and pooled effects with $95 \% \mathrm{Cl}$ ) regarding the risk of selected neurotoxicity of fatigue (A, random-effects model), headache (B, fixedeffects model), dysgeusia (C, random-effects model), vertigo (D, fixed-effects model), paresthesia (E, fixed-effects model), anxiety or malaise (F, random-effects model) and peripheral neuropathy (G, random-effects model) associated with nivolumab versus controls.

Abbreviations: RR, risk ratio; $\mathrm{Cl}$, confidence interval; $d f$, degree of freedom; $\mathrm{M}-\mathrm{H}$, Mantel-Haenszel.

\section{Discussion}

In the light of our present knowledge, this analysis is the most updated one to provide an evaluation of the neurotoxicities, including fatigue, headache, dysgeusia, vertigo, paresthesia, anxiety or malaise and peripheral neuropathy, in cancer patients treated with nivolumab. This meta-analysis summarized 6 Phase III randomized clinical trials, demonstrating that nivolumab did not cause increased or decreased risks of fatigue, headache, dysgeusia, vertigo and anxiety or malaise and was associated with decreased risks of paresthesia and peripheral neuropathy as compared with controls.

Normally, T-cells have the capacity to attack cancer cells; meanwhile, T-cells have to be regulated properly through certain inhibitory checkpoints to be controlled to not attack normal cells and normal tissues. ${ }^{17}$ According to this theory, inhibiting these checkpoints is likely to activate T-cells and lead them to have stronger anticancer responses. ${ }^{18}$ Currently, PD-1, as an inhibitory receptor, is one of the most topical, and its antagonist nivolumab has been developed as a US FDA-approved anticancer drug for melanomas, which is based on the findings of a mound of Phase II-III studies. ${ }^{6}$ Moreover, nivolumab has also been evaluated for its effects on anti-gastrointestinal cancer, prostate cancer and lung cancer. According to the National Cancer Institute (NCI), nivolumab is approved to be used alone or with other drugs to treat: 1) classical Hodgkin's lymphoma that has gotten worse after an autologous stem cell transplant and treatment with brentuximab vedotin; 2) melanoma that cannot be removed by surgery or that has metastasized (spread to other parts of the body); 3) NSCLC that has metastasized. It is used in patients whose disease got worse during or after treatment with platinum chemotherapy; and 4) renal cell carcinoma (a type of kidney cancer) that is advanced. It is used in patients who have already received angiogenesis inhibitor therapy. Pembrolizumab is another drug that has been recently approved by the US FDA. Formerly known as MK-3475 or lambrolizumab, it is a high affinity humanized IgG4 monoclonal antibody targeting PD-1, having been approved to treat: 1) melanoma that cannot be removed by surgery or that has metastasized (spread to other parts of the body); 2) NSCLC that has metastasized and 3) squamous cell carcinoma of the head and neck that has metastasized or recurred (come back) in patients whose disease got worse during or after treatment with platinum chemotherapy. With respect to the differential activity of nivolumab and pembrolizumab, the former is a fully human IgG4 PD-1 immune checkpoint inhibitor antibody.

The nervous system adverse effects have been regarded as an emerging reason for treatment discontinuation or interruption in some studies. Although neurotoxicities are not common, at present, no effective methods have been developed to predict high-risk patients, necessitating careful monitoring of laboratory and clinical parameters. Neurotoxicities have also been reported in a number of other targeted anticancer therapeutics and have been linked to noncompliance with many of them..$^{8-13}$ In light of this, we combined PubMed, Google 
Scholar, Embase and CNKI databases to systematically analyze the correlations between the risks of fatigue, headache, dysgeusia, vertigo, paresthesia, anxiety or malaise and peripheral neuropathy and nivolumab. The results of our study demonstrated that when compared with controls, nivolumab did not statistically increase the risks of selected neurotoxicity of paresthesia and peripheral neuropathy. Therefore, although clinicians should be attentive to the side effects of nivolumab, in terms of nervous system side effects, nivolumab is safe.

On a contour-enhanced funnel plot, contours of statistical significance are overlaid on the funnel plot. Adding contours of statistical significance facilitates the assessment of whether the areas where studies exist are areas of statistical significance and whether the areas where studies are potentially missing correspond to areas of low statistical significance. Generally, if studies appear to be missing in areas of low statistical significance, then it is very possible that the asymmetry is due to publication bias. Conversely, if the areas where studies are perceived to be missing are areas of high statistical significance, then publication bias is not the cause of funnel asymmetry. ${ }^{19}$ In our meta-analysis, the funnel plot indicated no publication bias.

The results of our study should be interpreted with caution because the meta-analysis is at a study level, thus limiting variables at patient levels might not be worked out. So, we could not confirm if there are any additional potential risk factors correlated to developments of neurotoxicities.

\section{Conclusion}

Our analysis supported that the PD-1 inhibitor nivolumab did not cause increased or decreased risks of fatigue, headache, dysgeusia, vertigo and anxiety or malaise and was associated with decreased risks of paresthesia and peripheral neuropathy as compared with controls. These outcomes indicated that in terms of nervous system side effects, nivolumab is safe.

\section{Acknowledgments}

We would like to thank our colleagues at the Department of Neurosurgery, Peking Union Medical College Hospital, Chinese Academy of Medical Sciences and Peking Union Medical College. This study was supported by Peking Union Medical College Youth Research Funds (2016) (Project No 3332016010; Grant recipient: Xiangyi Kong) and Peking Union Medical College Graduate Student Innovation Fund (2015) (Project No 2015-1002-02-09; Grant recipient: Xiangyi Kong). The funders had no role in study design, data collection and analysis, decision to publish or preparation of the manuscript.

\section{Disclosure}

The authors report no conflicts of interest in this work.

\section{References}

1. Abdel-Rahman O. Immune checkpoints aberrations and gastric cancer; assessment of prognostic value and evaluation of therapeutic potentials. Crit Rev Oncol Hematol. 2016;97:65-71.

2. Momtaz P, Postow MA. Immunologic checkpoints in cancer therapy: focus on the programmed death-1 (PD-1) receptor pathway. Pharmacogenomics Pers Med. 2014;7:357-365.

3. Hamid O, Robert C, Daud A, et al. Safety and tumor responses with lambrolizumab (anti-PD-1) in melanoma. N Engl J Med. 2013;369(2): 134-144.

4. Lim TS, Chew V, Sieow JL, et al. PD-1 expression on dendritic cells suppresses CD8 T cell function and antitumor immunity. Oncoimmunology. 2016;5:e1085146.

5. Rajan A, Kim C, Heery CR, Guha U, Gulley JL. Nivolumab, antiprogrammed death-1 (PD-1) monoclonal antibody immunotherapy: role in advanced cancers. Hum Vaccin Immunother. 2016;12(9): 2219-2231.

6. Brahmer JR, Hammers H, Lipson EJ. Nivolumab: targeting PD-1 to bolster antitumor immunity. Future Oncol. 2015;11(9):1307-1326.

7. Brahmer JR, Tykodi SS, Chow LQ, et al. Safety and activity of antiPD-L1 antibody in patients with advanced cancer. N Engl J Med. 2012; 366:2455-2465.

8. Borghaei H, Paz-Ares L, Horn L, et al. Nivolumab versus docetaxel in advanced nonsquamous non-small-cell lung cancer. N Engl J Med. 2015; 373(17):1627-1639.

9. Brahmer J, Reckamp KL, Baas P, et al. Nivolumab versus docetaxel in advanced squamous-cell non-small-cell lung cancer. NEngl JMed. 2015; 373:123-135.

10. Larkin J, Chiarion-Sileni V, Gonzalez R, et al. Combined nivolumab and ipilimumab or monotherapy in untreated melanoma. NEngl J Med. 2015;373:23-34.

11. Motzer RJ, Escudier B, McDermott DF, et al. Nivolumab versus everolimus in advanced renal-cell carcinoma. $N$ Engl J Med. 2015;373: 1803-1813.

12. Robert C, Long GV, Brady B, et al. Nivolumab in previously untreated melanoma without BRAF mutation. $N$ Engl J Med. 2015;372(4): 320-330.

13. Weber JS, D'Angelo SP, Minor D, et al. Nivolumab versus chemotherapy in patients with advanced melanoma who progressed after anti-CTLA-4 treatment (CheckMate 037): a randomised, controlled, open-label, phase 3 trial. Lancet Oncol. 2015;16(4):375-384.

14. National Cancer Institute[database on internet]. Bethesda, MD: National Institutes of Health. Available from: https://www.eortc.be/services/doc/ ctc/CTCAE_4.03_2010-06-14_QuickReference_5x7.pdf. Accessed February 16, 2017.

15. Dickersin K, Berlin JA. Meta-analysis: state-of-the-science. Epidemiol Rev. 1992;14:154-176.

16. Higgins JP, Thompson SG, Deeks JJ, Altman DG. Measuring inconsistency in meta-analyses. BMJ. 2003;327:557-560.

17. Swanson MS, Sinha UK. Rationale for combined blockade of PD-1 and CTLA-4 in advanced head and neck squamous cell cancer-review of current data. Oral Oncol. 2015;51:12-15.

18. Topalian SL, Hodi FS, Brahmer JR, et al. Safety, activity, and immune correlates of anti-PD-1 antibody in cancer. $N$ Engl J Med. 2012;366: 2443-2454.

19. Peters JL, Sutton AJ, Jones DR, Abrams KR, Rushton L. Contour-enhanced meta-analysis funnel plots help distinguish publication bias from other causes of asymmetry. J Clin Epidemiol. 2008;61(10):991-996.

20. Moher D, Liberati A, Tetzlaff J, Altman DG. The PRISMA Group (2009). Preferred reporting items for systematic reviews and metaanalyses: the PRISMA statement. PLoS Med. 6(7):e1000097. 


\section{Publish your work in this journal}

OncoTargets and Therapy is an international, peer-reviewed, open access journal focusing on the pathological basis of all cancers, potential targets for therapy and treatment protocols employed to improve the management of cancer patients. The journal also focuses on the impact of management programs and new therapeutic agents and protocols on

patient perspectives such as quality of life, adherence and satisfaction. The manuscript management system is completely online and includes a very quick and fair peer-review system, which is all easy to use. Visit http://www.dovepress.com/testimonials.php to read real quotes from published authors.

Submit your manuscript here: http://www.dovepress.com/oncotargets-and-therapy-journal 